\title{
La figure de l'entrepreneur textile dans le Maine : l'exemple de la famille Véron (1684-v.1750)
}

\section{Alexis Goethal}

\section{Q OpenEdition \\ 1 Journals}

Édition électronique

URL : http://journals.openedition.org/abpo/3849

DOI : $10.4000 / a b p o .3849$

ISSN : 2108-6443

Éditeur

Presses universitaires de Rennes

Édition imprimée

Date de publication : 12 juillet 2018

Pagination : 123-143

ISBN : 978-2-7535-7604-9

ISSN : 0399-0826

Référence électronique

Alexis Goethal, « La figure de l'entrepreneur textile dans le Maine : l'exemple de la famille Véron (1684v.1750) », Annales de Bretagne et des Pays de l'Ouest [En ligne], 125-2 | 2018, mis en ligne le 12 juillet 2020, consulté le 06 janvier 2021. URL : http://journals.openedition.org/abpo/3849 ; DOI : https:// doi.org/10.4000/abpo.3849 


\title{
La figure de l'entrepreneur textile dans le Maine : l'exemple de la famille Véron (1684-v.1750)
}

\author{
Alexis GOETHAL \\ Étudiant de master 1, université du Maine (direction : Benoît Musset)
}

Au début du XVIII ${ }^{\mathrm{e}}$ siècle, Le Mans était une ville provinciale de taille moyenne (environ 15000 habitants) dont près du tiers de la population vivait de la fabrication et du commerce de l'étamine. Il s'agit d'une étoffe de laine fine, très prisée par les communautés religieuses. Une famille serait à l'origine de l'invention de ce tissu et du développement de ce commerce qui tira l'essor de toute la région du Mans : les Véron. Le membre le plus célèbre de cette famille n'est autre que François Véron de Forbonnais (17221800), l'économiste physiocrate auteur des Éléments du commerce (1754). La réussite de François de Forbonnais contribua à focaliser l'attention des historiens et érudits locaux sur la généalogie de l'homme, bien souvent en glorifiant la famille. En effet, il semble que l'arrière-grand-père de l'économiste manceau, Jean Véron (1615-1689) soit l'inventeur de l'étamine du Mans. Son grand-père, Guillaume Véron l'aîné (1656-1723), serait quant à lui un inventeur hors norme, tandis que ses trois fils, Guillaume Véron le jeune (1692-1741), Véron-Duverger (1695-1780) le père de Forbonnais, et Véron de La Croix (1704-1758), seraient responsables du développement de la commercialisation de l'étamine du Mans à l'international.

Nous sommes donc face à une véritable dynastie entrepreneuriale, paradoxalement très peu étudiée. Pourtant, cette famille est la principale animatrice de la vie économique mancelle durant la première moitié du XVIII ${ }^{\mathrm{e}}$ siècle et demeure connue des Manceaux par sa maison familiale, située en centre-ville, ornée d'une sirène, symbole de la prospérité du commerce maritime lié à l'étamine. Seuls les travaux de François Dornic, L'industrie textile dans le Maine et ses débouchés internationaux (1650-1815), datant de 1955, se focalisèrent sur la question du textile dans la ville du Mans et abordèrent la famille Véron. Toutefois, cette étude se cantonnait au cas de Guillaume Véron l'aîné dans la plus pure tradition schumpétérienne. Il convient donc d'apporter une relecture historiographique, et d'ouvrir 
de nouvelles perspectives en ce qui concerne les entrepreneurs textiles manceaux, à partir de l'exemple des Véron.

\section{Illustration 1 - généalogie simplifiée de la famille Véron}

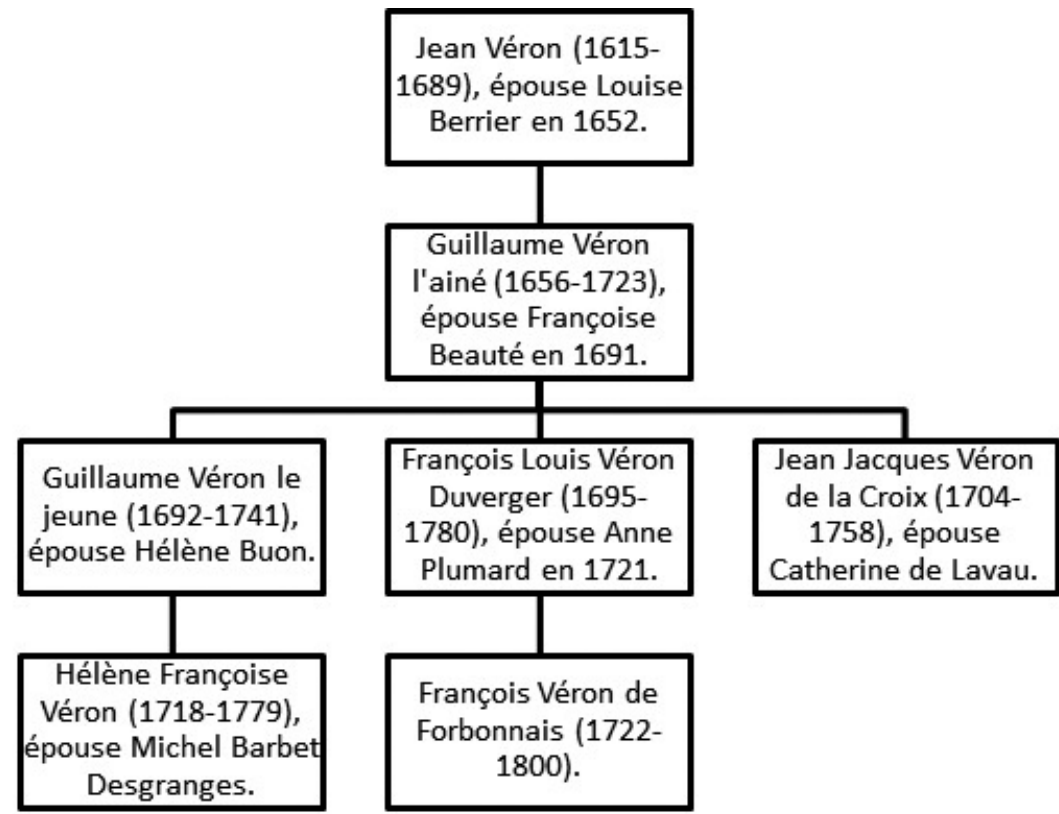

Les premiers à penser le concept d'entrepreneur sont les économistes classiques. L'irlandais Richard Cantillon (1680-1734) va en proposer une première définition, précisée ensuite par Jean-Baptiste Say (1767-1832) ${ }^{1}$, principal représentant du courant économique classique en France. Dans un premier temps, à défaut d'intéresser les historiens, la figure de l'entrepreneur fascine les premiers économistes. On cherche alors simplement à définir ce nouvel acteur que l'on devine jouer un rôle de premier plan. Mais ce n'est vraiment qu'avec les travaux de Joseph Alois Schumpeter $(1883-1950)^{2}$ que la figure de l'entrepreneur va apparaitre sur le devant de la scène, se dissociant par-là même de la figure du capitaliste. Schumpeter va s'atteler à définir la figure d'un entrepreneur qui entre dans une logique plus large, visant à expliquer les cycles économiques ${ }^{3}$. Ainsi, il fait de cet acteur l'agent principal de la célèbre destruction créatrice qui engendre les mutations successives du capitalisme. Ces trois économistes forgent donc la figure de l'entrepreneur, le définissent de plus en plus précisément

1. RaviX, Joël Thomas, "Jean-Baptiste Say et l'entrepreneur : la question de la filiation avec Cantillon et Turgot ", Innovations, n 45, 2014, p. 59-76.

2. Schumpeter, Joseph, Théorie de l'évolution économique, Paris, Dalloz, 1935.

3. LAPIED, André, SWATON, Sophie, « Le cycle de la conjoncture chez Schumpeter : éternel retour du même? ", Revue de philosophie économique, vol. 15, 2014, p. 17-47. 
avant de l'inclure dans une théorie plus large. Karl Marx fait également de l'entrepreneur, ou plutôt du producteur faisant commerce de ses produits, l'agent responsable du passage à une société capitaliste ${ }^{4}$. Les historiens ne sont pas restés insensibles à ces travaux, notamment au moment où l'histoire économique était en vogue. En témoigne, le développement de la Business History ${ }^{5}$ outre-Atlantique dans les années 1960. Dans l'Hexagone, c'est Fernand Braudel qui renversa la perspective : "Je ne crois pas que J. Schumpeter ait raison de faire de l'entrepreneur le deus ex machina. Je crois que c'est le mouvement d'ensemble qui est déterminant ${ }^{6}$. " En effet, si pour l'économiste autrichien, l'entrepreneur était l'agent moteur du capitalisme en marche, un entrepreneur idéal ${ }^{7}$, pour Fernand Braudel il serait plutôt une conséquence du contexte économique global. Pour lui, un secteur va être précurseur des évolutions à venir tant son poids est important au cours du XVIII ${ }^{\mathrm{e}}$ siècle : le textile ${ }^{8}$. Il note que c'est précisément dans ce secteur que les premiers entrepreneurs vont apparaître, alliant gestion de la production et commercialisation des produits.

On cerne d'ailleurs de mieux en mieux les contours de cette élite négociante avec les travaux de Serge Chassagne ${ }^{9}$ ou d'André Lespagnol qui étudia l'élite marchande malouine ${ }^{10}$. Bordeaux ${ }^{11}$ et Marseille ${ }^{12}$ disposent chacune de leur monographie commerciale analysant systématiquement les élites marchandes et entrepreneuriales. Toutefois, nous pouvons noter que les études ont longtemps concerné principalement les villes portuaires. Il faudra attendre la remarquable étude de Serge Chassagne sur Oberkampf pour voir l'étude spécifique d'un entrepreneur au cours du XVIII ${ }^{\mathrm{e}}$ siècle $^{13}$. La définition progressive des entrepreneurs du secteur textile est, par la suite, approfondie dans les monographies régionales consacrées aux manufactures textiles, à l'image de celle de Gérard Gayot ${ }^{14}$ sur les draps de Sedan ou celle de Jean-Michel Minovez ${ }^{15}$ sur les draps du Languedoc. Claude Marquié

4. GAYOT, Gérard, Les draps de Sedan 1646-1870, Paris, ehess, 1998, p. 11.

5. ChAndler, Alfred, La main visible des managers, une analyse historique, Paris, Economica, 1988.

6. BRAUDEL, Fernand, La dynamique du capitalisme, Paris, Armand Colin, 1985, p. 67.

7. BouTILlER, Sophie, Uzunidis, Dimitri, «L'empreinte historique de la théorie de l'entrepreneur. Enseignements tirés des analyses de Jean-Baptiste Say et de Joseph Aloïs Schumpeter ", Innovations, $n^{\circ} 45,2014$, p. 115.

8. BRAUDEL, Fernand, Civilisation matérielle, économie et capitalisme, Paris, Armand Colin, 1979.

9. Chassagne, Serge, Le coton et ses patrons, France, 1760-1840, Paris, EHESS, 1991.

10. Lespagnol, André, "Messieurs de Saint-Malo ": une élite négociante au temps de Louis XIV, Rennes, PUR, 1997 (1990).

11. BuTEL, Paul, Les négociants bordelais, l'Europe et les Îles au XVIII siècle, Paris, AubierMontaigne, 1974.

12. CARRIERE, Charles, Négociants marseillais au XVIII siècle. Contribution à l'étude des économies maritimes, Marseille, Institut historique de Provence, 1973.

13. ChaSSAGNE, Serge, Oberkampf, un grand patron au siècle des Lumières : l'inventeur de la toile de Jouy, Aubier, 2015 (1980).

14. GAYOT, Gérard, Les draps de Sedan, 1646-1870, Paris, EHESS, 1998.

15. Minovez, Jean-Michel, La Puissance du Midi. Les draperies de Colbert à la Révolution, Rennes, PUR, 2012. 
mena, quant à lui, une étude plutôt socio-économique du groupe des marchands fabricants carcassonnais dès les années $1990^{16}$. Ces études mêlent souvent aspects social, économique et histoire des techniques. C'est au début des années 1980 qu'Hélène Vérin s'attela à définir précisément l'évolution du terme d'entrepreneur ${ }^{17}$. Puis, à la suite de Liliane Hilaire-Perez ${ }^{18}$, des ouvrages s'intéressèrent au rapport entre ces hommes et la technique, l'innovation. Apparaît alors la figure d'un entrepreneur réalisant le lien entre le progrès scientifique des intellectuels et les ouvriers qui mettent en œuvre les nouveaux procédés ${ }^{19}$.

Nous procéderons donc à une relecture historiographique à partir du cas de Guillaume Véron avant d'aborder le rôle de la famille dans l'entreprise ainsi que le statut social de l'entrepreneur.

\section{Guillaume Véron : un entrepreneur schumpetérien?}

\section{De la position classique...}

Depuis 1669 et les règlements sur la draperie qui constituent l'acte fondateur du colbertisme dans le domaine textile, on cherche à augmenter la qualité de la production française afin de concurrencer les manufactures étrangères ${ }^{20}$. Ces considérations touchent particulièrement les secteurs de l'industrie du luxe. Les étamines du Mans sont concernées puisqu'elles sont très prisées pour leur finesse par les gens de robe. Le pouvoir royal accordait des privilèges royaux aux marchands ou fabricants dont l'ouvrage était réputé le meilleur. Le Dictionnaire de Furetière (1690) nous offre une définition du privilège d'Ancien Régime : " avantage particulier dont jouit une personne à l'exclusion de plusieurs [beaucoup] d'autres, qui lui vient par les bienfaits de son souverain ${ }^{21} "$.

Au terme d'une première action judiciaire (1695-1699) faisant suite à la réception de Guillaume Véron dans la communauté des marchands merciers ${ }^{22}$, il obtint un privilège royal. Le jeune marchand n'entendait pas renoncer au dégrais des étamines qu'il exerçait illégalement dans son atelier. Pourtant, un arrêt rendu par le conseil du roi le 17 septembre 1697 était venu confirmer que les fabricants étaient les seuls à pouvoir lustrer, dégraisser, dégorger et apprêter les étamines, au détriment des

16. MARQuIE, Claude, L'industrie textile carcassonnaise au XVII ${ }^{e}$ siècle. Étude d'un groupe social : les marchands-fabricants, Carcassonne, Sociétés d'études scientifiques de l'Aude, 1993.

17. Verin, Hélène, Entrepreneurs, entreprise. Histoire d'une idée, Paris, PUF, 1982.

18. HilaIRE-PEREZ, Liliane, L'invention technique au siècle des Lumières, Paris, Albin Michel, 2000 .

19. VERIN, Hélène, La gloire des ingénieurs. L'intelligence technique du XVI siècle au XVIII ${ }^{e}$ siècle, Paris, Albin Michel, 1993.

20. STANZIANI, Alessandro (dir.), La qualité des produits en France (XVIII ${ }^{e}-X X^{e}$ siècles), Paris, Belin, 2003, p. 70.

21. BELY, Lucien (dir.), Dictionnaire de l'Ancien Régime, Paris, PUF, 1996, " privilège ".

22. Arch. dép. de la Sarthe, 5 E 132, 19 mars 1712. 
marchands. Une campagne débuta alors visiblement auprès du lieutenant général du Mans, Levayer, afin de prouver par des témoignages de marchands locaux et étrangers que Guillaume Véron fabriquait bien les meilleures étamines de la ville, grâce au moulin à fouler de son invention. L'affaire fut finalement portée au conseil du roi le 23 juin 1699. Les sergers y réclamaient la réaffirmation de leur monopole sur le dégrais, lustrage et foulage ainsi que sur le cardage des étamines, au détriment des marchands dont Guillaume Véron faisait partie, sous peine de 3000 livres d'amende ${ }^{23}$. Guillaume Véron demanda quant à lui l'autorisation de se servir de son moulin pour apprêter les étamines avant et après la teinture, et que l'on cesse de troubler son commerce pour ce motif sous peine de 15000 livres d'amende. Finalement, le conseil du roi, sur l'avis du sieur Thomas Hue de Miromesnil, intendant de la généralité de Tours, ainsi que de Phélypeaux de Ponchartrain, contrôleur général des finances, trancha plutôt en faveur de Guillaume Véron l'aîné. Le droit de fouler, dégorger, apprêter les étamines sur la rivière en vertu de l'arrêt de 1697 fut confirmé aux sergers. On note bien dans la décision du conseil l'ambiguïté puisque cette réaffirmation des droits de l'arrêt de 1697 ne concerne pas les moulins. Les marchands peuvent donc, techniquement, continuer de fouler, dégraisser et apprêter les draps en utilisant des moulins. C'est le cas de Guillaume Véron, puisque l'arrêt " permet audit Guillaume Véron de continuer sa vie durant de se servir du moulin qu'il a inventé et fait construire dans sa maison pour fouler, laver, degraisser et aprester avant et après la teinture les estamines dont il fera commerce; sauf ausdits tondeurs, et ausdits sergers de faire construire des semblables moulins si bon leur semble ". À ce stade, Guillaume Véron dispose donc d'un avantage sur les autres marchands drapiers, ses confrères, puisqu'il est le seul à pouvoir apprêter les étamines. On peut donc supposer qu'il est ainsi plus compétitif, puisqu'il supprime des intermédiaires.

Une seconde étape est franchie lorsque, non content d'avoir obtenu le privilège, Guillaume Véron l'aîné souhaita le transmettre à ses enfants ${ }^{24}$. Il ne s'opposait alors plus seulement à la communauté des sergers mais également à celle des marchands. Tous s'accordaient pour remettre en cause le privilège de Guillaume Véron. Une lettre de Bernard Chauvelin, maître des requêtes, intendant de la généralité de Tours, nous montre une prise de parti en faveur de Guillaume Véron, le 30 avril $1712^{25}$. Il y explique que le privilège de 1697 a été très bénéfique pour le commerce des étamines et que, par conséquent, il convient de le transmettre à l'aîné des enfants Véron. Il nous apprend que Véron, ayant élevé cinq de ses enfants dans le commerce des étamines, devrait privilégier la transmission du privilège seulement à l'aîné des enfants dans le but de ne pas créer de trop fortes tensions avec les sergers, le privilège pouvant ensuite se transmettre comme n'importe

23. Arch. nat., E 688 B, 13 juin 1699.

24. Arch. dép. de la Sarthe, 5 E 132, 19 mars 1712.

25. Arch. nat., $\mathrm{F}^{12} 1372,30$ avril 1712. 
quel bien au sein d'une même famille ${ }^{26}$. Finalement le conseil du commerce du 10 mars 1713 trancha en faveur de Guillaume Véron l'aîné et lui concéda le droit de transmettre son privilège à l'enfant de son choix ${ }^{27}$. Dans son testament, Guillaume Véron l'aîné affirmat vouloir que Guillaume Véron le jeune, son fils aîné, en soit le bénéficiaire ${ }^{28}$; mais le 21 juillet 1721 , le testament fut modifié au profit de François Véron Duverger, son second fils ${ }^{29}$.

Ces éléments constituaient pour François Dornic la démonstration éclatante que Guillaume Véron devait lutter seul contre les communautés de métier. Il écrivit même que cet exemple " jette une vive lumière sur l'esprit de routine des anciennes communautés de métiers, Guillaume Véron, le fils du créateur de l'étamine camelotée, lui-même inventeur, dressé seul [...] contre les représentants des deux communautés drapières, qui n'ont guère à lui opposer que de l'envie et des articles de statuts ${ }^{30}$ ". Il fait ainsi de Guillaume Véron, dans la logique schumpéterienne qui est la sienne, un entrepreneur qui vient remettre en cause l'ordre établi et il interprète ces procès comme une volonté de résistance au progrès de la part des institutions.

\section{... à une relecture historiographique}

Pourtant, il nous semble que la recherche du privilège royal de la part de Guillaume Véron relève davantage d'une logique commerciale. Nous ne sommes pas ici dans le cas de l'entrepreneur qui remet en cause l'ordre social. D'ailleurs, ce type d'entrepreneur est une fiction puisqu'il entreprend et innove de façon totalement désintéressée. Or, il apparaît dans les procès-verbaux de 1712 que le véritable objectif de la mise en service de la machine de l'invention de Guillaume avait été " qu'il luy en coustat moins à aprester ses marchandises ${ }^{31}$ ". Dans la même logique, obtenir un privilège permet à l'entrepreneur manceau de bénéficier d'un avantage significatif vis-à-vis de ses concurrents.

La tradition historiographique oppose souvent deux visions : la première, dans la veine de celle de la société de corps, idéalise les communautés et fait d'elles une institution indispensable au bon ordonnancement de la société; la seconde, plus libérale, dénonce l'inefficacité économique de ce système que l'on décrit souvent comme rongé par les rigidités et les blocages $^{32}$. C'est dans la seconde vision que va s'inscrire Dornic lorsqu'il analyse le rapport des Véron à la communauté de métier, fortement influencé

26. HilaIRE-PerEz, Liliane, L'invention technique..., op. cit., p. 115.

27. DoRnIC, François, L'industrie textile dans le Maine et ses débouchés internationaux (1650-1815), Le Mans, Édition Pierre-Belon, 1955, p. 28.

28. Arch. dép. de la Sarthe, 4 E 25 109, 25 septembre 1713.

29. DoRnic, François, L'industrie textile..., op. cit., p. 28.

30. Ibid., p. 27.

31. Arch. dép. de la Sarthe, 5 E 132, 19 mars 1712.

32. KAPLAN, Steven, MINARD, Philippe, La France malade du corporatisme? XVIII $-X X^{e}$ siècles, Paris, Belin, 2004, p. 39. 
par l'idée des Lumières selon laquelle elle constituerait un frein à l'innovation $^{33}$. N'oublions pas que Guillaume Véron a été, après ces démêlés, garde juré de la communauté (1720), ce qui nous prouve bien qu'il n'est pas dans le rejet de l'institution, dans l'opposition frontale avec ce qui constituerait un frein à son commerce. La communauté apparaît ici plutôt comme un moyen de domination sociale et économique que comme un véritable organisme de production de règles qui seraient hostiles aux Véron ${ }^{34}$. D'ailleurs, ses enfants ont été tout au long du XVIII ${ }^{\mathrm{e}}$ siècle appelés aux responsabilités au sein de la communauté des marchands.

\section{Un héritage technique}

Un moulin avait été mis au point par Guillaume Véron l'aîné quelques années plus tôt. Avec ce moulin, il aurait fait " fabriquer des étamines plus belles et plus fines que l'on n'avoit encore fait, il trouva le moyen de leur donner la finesse et le beau grain que nous leur voyons et poussa cet art jusqu'à la perfection ${ }^{35}$ ". Ici, c'est par l'invention de son moulin à fouler, dégorger et apprêter les étamines qu'il aurait innové et contribué à donner aux étamines du Mans une plus grande perfection. Pour F. Dornic, il ne faisait pas de doute que Guillaume Véron était bien l'inventeur d'un tel moulin ${ }^{36}$ et que c'était seulement la jalousie qui faisait contester son invention. Or, il nous semble que des arguments intéressants sont mobilisés par les marchands s'opposant à Guillaume Véron l'aîné en 1712. En effet, ces derniers nous disent que la fabrication du moulin de Guillaume Véron n'est aucunement un secret, et est connue de tous. D'ailleurs, le privilège exclusif dont jouit notre négociant ne mentionne pas d'interdiction pour les autres marchands de construire pareil moulin. Le cœur du problème semble davantage être d'ordre financier que technique. En effet, les marchands nous disent que le secret de ce moulin n'est pas " inconnu aux autres sargers et [qu']il n'y a aucun maistre qui ne scust en faire construire un égail ${ }^{37}$ ", il apparaît ici que le vrai problème est, plutôt qu'une ignorance du fonctionnement technique de la machine en elle-même, une incapacité à financer sa construction. Les sergers remarquent d'ailleurs que si Guillaume Véron l'aîné a réussi dans son commerce, ce n'est que parce qu'il possédait un réseau plus important et des financements en quantité suffisante. Ainsi, nous nous éloignons de la figure d'un self made man du siècle des Lumières ${ }^{38}$. Les marchands, quant à eux, affirmaient que la machine de Guillaume Véron n'était qu'un moulin semblable à ceux des autres marchands, et qu'il cherchait en réalité à obtenir un monopole en le faisant

33. KAPLAN, Steven, La fin des corporations, Paris, Fayard, 2001, p. 16.

34. KAPLAN, Steven, MinARD, Philippe, La France malade du corporatisme?..., op. cit., p. 50.

35. Médiathèque Louis Aragon, Le Mans, Mémoires de Négrier de la Crochardière, t. II, p. $180-181$.

36. DoRnic, François, L'industrie textile..., op. cit., p. 27.

37. Arch. dép. de la Sarthe, 5 E 132, 19 mars 1712.

38. HilaIRE-PEREZ, Liliane, L'invention technique ..., op. cit., p. 169. 
passer pour une invention nouvelle et que sans "l'expérience de tous les maistres de lad communauté " la technique pour apprêter les étamines n'aurait pas été mise au point. On voit donc ici que l'apport sur le plan technique est loin d'être incontestable.

Du fonctionnement de ce moulin nous ne savons pas grand-chose, les arrêts du conseil du roi et les pièces des procès parlent d'une machine. Il nous semble que ce moulin fut sans doute mis en place vers $1690^{39}$. Nous pouvons également déduire de l'arrêt de $1699^{40}$ que ce moulin n'utilise pas la force hydraulique puisque, à cette époque, dans la paroisse du Pré où il résidait, seuls les sergers étaient autorisés à utiliser la force de la rivière. Les écrits de Négrier de la Crochardière nous fournissent une précision cruciale : " il inventa une machine qui est une espece de moulin qu'on fait tourner avec un cheval, pour fouler et dégorger les étamines ce qui avec les soins qu'il se dona pour le degrais a rendu les etamines si belles que par toute l'europe ou ce commerce s'est étendu, on nomme ses belles étamines des veronnes ${ }^{41}$ ". On voit ici que pour concevoir une telle machine il devait faire preuve d'une grande ingéniosité et posséder un capital technique très important. Il est par ailleurs intéressant de constater que ce capital se transmet de génération en génération. En témoigne le cas de son fils aîné, Guillaume Véron le jeune, chez qui l'on trouve une salle visiblement dédiée à l'entretien et la conception de pièces : "Sommes entrée dans la chambre du laboratoire autour ou travailloit led défunt $\mathrm{S}^{\mathrm{r}}$ Véron $[\ldots]^{42}$. " On y trouve alors énormément de matériel de menuiserie destiné probablement à entretenir des machines, à savoir : une hache, deux scies, deux roues de moulins, quatorze mèches à percer, sept limes à fer et trois limes à bois, six ciseaux à bois... Ainsi, Guillaume Véron apparaît davantage comme un " améliorateur ${ }^{43}$ " que comme un réel inventeur. Il semble bien que l'octroi du privilège consacre une stratégie entrepreneuriale visant à accaparer l'usage d'un procédé connu de tous.

\section{Entre le cour et la raison : entreprendre collectivement}

\section{Une alliance capitale}

Étudier l'entrepreneur au siècle des Lumières c'est également se soucier des stratégies mises en place dans le but de développer l'entreprise familiale. À cet égard, le mariage de 1721 entre François Louis Véron Duverger et Anne Plumard est riche d'enseignements. En effet, les Plumard étaient l'une des dix familles les plus puissantes de la ville du Mans et leur assise financière était incontestable. Le chef de famille, Louis Plumard l'aîné ainsi

39. Arch. dép. de la Sarthe, 4 E 25 12, 7 janvier 1690.

40. Arch. nat., E 688 B, 13 juin 1699.

41. Médiathèque Louis Aragon. Mémoires de Négrier de la Crochardière, t. II, p. 182-183.

42. Arch. dép. de la Sarthe, 28 J 358, 23 octobre 1744.

43. MinARD, Philippe, La fortune du colbertisme. Etat et industrie dans la France des Lumières, Paris, Fayard, 1998, p. 228. 
que ses deux fils, Louis Plumard le jeune et Jacques Plumard de Rieux, étaient parmi les acteurs principaux du commerce de l'étamine. Les relations entre les deux familles étaient anciennes : d'une part, au sein de la communauté des marchands, de l'autre au collège de l'Oratoire où François Louis Duverger côtoya Louis Plumard le jeune ${ }^{44}$. Il était alors intéressant, en se fondant sur un critère purement financier, de contracter une alliance avec cette famille afin de mutualiser les réseaux commerciaux et d'alimenter en capitaux le très gourmand et très lent processus de fabrication des étamines.

De plus, François Dornic imaginait que ce mariage pouvait constituer une suite du procès de 1712. En effet, on notait la présence de Louis Plumard l'aîné parmi les marchands contestant le privilège de Guillaume Véron sur le procès-verbal de 1712. Le mariage constituerait probablement un compromis entre les deux familles. Nous avons désormais confirmation que cette intuition était juste. En effet, le contrat de mariage dont ne disposait pas François Dornic en son temps, nous montre bien que la transmission du privilège était une condition mise à l'alliance des deux familles ${ }^{45}$. On voit d'ailleurs qu'à peine dix jours après la signature du contrat de mariage, François Louis Duverger devient l'héritier du privilège royal qui était au départ concédé à son frère aîné Guillaume le jeune. Louis Plumard l'aîné ${ }^{46}$ se retire du commerce des étamines, tout comme Guillaume Véron l'aîné ${ }^{47}$. Guillaume Véron le jeune renonce par la suite à ses droits dans la communauté des marchands ${ }^{48}$ ainsi qu'à son rôle d'enseigne de milice bourgeoise de la ville du Mans en faveur de son frère François Louis ${ }^{49}$. Il apparaît donc comme le grand gagnant de ce remaniement familial dans lequel le privilège tient le premier rôle. Nous sommes donc une fois de plus ici face à un tournant dans l'expansion du commerce des Véron. On assiste par la suite à une spécialisation familiale, Jacques Plumard de Rieux s'installant comme armateur à Nantes et les Véron gérant la production au Mans ${ }^{50}$.

Cette alliance permet, de plus, de faire baisser mécaniquement le risque de pertes lié au grand commerce, une des solutions consistant à varier les espaces géographiques afin de parer à une conjoncture locale difficile. Le premier cas rencontré est celui du mariage de Guillaume Véron avec Mathurine Hervé, de Bonnétable, qui nous est connu par un contrat de mariage passé le 4 octobre $1684^{51}$. Or, il se trouve que ce bourg constitue à la fin $\mathrm{du} \mathrm{XVII}^{\mathrm{e}}$ siècle un important centre étaminier. On sait par exemple que les Plumard, grande famille du négoce manceau, sont originaires de

44. Arch. dép. de la Sarthe, C ADDITIF 6.

45. BnF, fond Chappée 121, 12 juillet 1721.

46. Arch. dép. de la Sarthe, 5 E 129, 23 mars 1729.

47. Arch. dép. de la Sarthe, 4 E 25 117, 14 août 1721.

48. Arch. dép. de la Sarthe, 4 E 109, 14 août 1713.

49. Arch. dép. de la Sarthe, 4 E 25 115, 7 février 1719.

50. MEYER, Jean, L'armement nantais dans la deuxième moitié du XVIII siècle, Paris, EHESS, 1999, p. 45.

51. Arch. dép. de la Sarthe, 4 E 25 6, 4 octobre 1684. 
Tuffé près de Bonnétable et viennent s'établir au Mans seulement à la fin du $\mathrm{XVII}^{\mathrm{e}}$ siècle $^{52}$. Il est intéressant de remarquer qu'à la même période ces deux familles constituèrent une alliance Le Mans-Bonnétable. Il nous semble évident que Bonnétable constituait à la fin du XVII ${ }^{\mathrm{e}}$ et au début du XVIII ${ }^{\mathrm{e}}$ siècle un important relais pour les flux de bovins, de toiles et de menues denrées convergeant vers Paris. C'est d'ailleurs la même logique qui semble pousser au mariage de la fille aînée de François Louis Véron-Duverger avec le sieur Besnard de la Touche, négociant à Nogent-le Rotrou ${ }^{53}$. Cette ville était également un important centre de production étaminier, et ce depuis les années $1660^{54}$. À la fin du XVII ${ }^{\mathrm{e}}$ siècle la fabrication des étamines occupait d'ailleurs $21 \%$ des taillables dans la région de Nogent ${ }^{55}$. La ville représentait bien sûr un point stratégique en termes de réseau commercial afin de s'étendre vers Paris. On remarque que les mariages des filles de la famille étaient souvent l'occasion de diversifier les espaces géographiques ou les cercles professionnels ${ }^{56}$.

\section{L'importance du travail féminin}

Si le choix des épouses était crucial pour les entrepreneurs textiles, c'est également parce qu'en plus d'un apport de capitaux non négligeable par les dots, elles jouaient sans doute un rôle plus important que ce qu'on a bien longtemps cru. La question du travail féminin a longtemps été reléguée au second plan, presque éludée, comme si ce travail était d'une moindre importance, que ce soit quantitativement ou qualitativement. Mais depuis une trentaine d'années, tiré par l'essor des gender studies, le rôle des femmes dans l'économie tend à être mieux connu. Toutefois, si la place des femmes dans les processus d'industrialisation n'est plus à démontrer, celle qu'elles occupaient dans les économies préindustrielles est beaucoup plus floue. La faute en revient pour l'essentiel à des sources qui en disent bien souvent trop peu sur ce sujet. L'historiographie s'accordait jusqu'à présent à présenter le travail féminin comme essentiellement domestique. La Révolution industrielle serait venue mettre fin à cette répartition sexuée du travail ${ }^{57}$. Pourtant, la place des femmes dans la proto-industrie textile est loin d'être négligeable, notamment pour les

52. FLEURY, Gabriel, François Veron de Forbonnais : sa famille, sa vie, ses actes, ses œuvres (1722-1800), Mamers, Fleury, 1915, p. 19.

53. CAILly, Claude, "Deux familles de négociants d'étamines au Perche : les Margonne et les Buttet. Étude prosopographique ", Annales de Bretagne et des Pays de l'Ouest, n 119 , 2012, p. 117-144.

54. CAILLY, Claude, Mutation d'un espace proto-industriel : Le Perche aux XVIII ${ }^{e}$-XIX ${ }^{e}$ siècles, Ceton, Fédération des amis du Perche, 1993.

55. Desseix, Patrick, " Toiles et Étamines : l'axe Le Mans-Nogent sous l'Ancien Régime », Cénomane, no 9, 1983, p. 9-11.

56. LABORIE, Philippe, Approche de deux familles de grands négociants du Mans sous l'Ancien Régime. Les Fréart et les Garnier, mémoire de maîtrise en histoire sous la direction d'Anne Fillon et Jean-Marie Constant, université du Maine, 1990, p. 57.

57. Dousset, Christine, "Commerce et travail des femmes à l'époque moderne en France ", Les Cahiers de Framespa [en ligne], nº 2, 2006, consulté le 27 février 2017. 
différentes étapes du tissage (bobinage, dévidage et ourdissage ${ }^{58}$ ). En Angleterre, les travaux de Maxine Berg ${ }^{59}$ mettent bien en évidence le rôle central des femmes et des enfants dans le processus d'industrialisation et dans l'industrie manufacturière du XVIII ${ }^{\mathrm{e}}$ siècle. Au Mans, il en va de même : la fabrication des étamines occupe environ 12000 ouvriers à la veille de la Révolution, dont 9000 femmes et enfants ${ }^{60}$.

Toutefois, évaluer la place de ces femmes reste difficile pour nous ${ }^{61}$, même si les sources fiscales nous donnent quelques indices, puisqu'elles laissent apparaître les veuves. Ainsi, en $1760^{62}$, nous avions 14,4\% de veuves dans la communauté des marchands du Mans. Ces femmes apparaissent seules dans le relevé. Cependant, on peut penser qu'en réalité elles travaillent avec un fils, un beau-frère ou un gendre puisque, comme le remarque Christine Dousset, la femme a souvent un rôle de transmission du patrimoine. Elle assure en quelque sorte l'intermède entre deux hommes : un mari décédé et un fils trop jeune pour reprendre l'affaire familiale par exemple. Il est donc difficile de cerner le degré d'implication des femmes dans l'activité commerciale puisque le veuvage va constituer le seul moment où le travail des femmes va se retrouver institutionnalisé ${ }^{63}$. Mais se borner à l'étude des veuves comme c'est bien souvent le cas serait à notre sens une erreur. En effet, la famille apparaît comme une entité économique propre dans laquelle la femme tient une place de choix qu'il convient d'examiner plus en détail. De plus, la forte endogamie du milieu des négociants manceaux nous laisse présager une implication assez forte des femmes dans la vie économique locale.

Lors des nombreuses absences des négociants, celles-ci devaient les remplacer comme en témoigne, une procuration signée de la main de Jean Véron de la Croix ${ }^{64}$. Le négociant manceau l'établit le 15 mai 1739 devant un notaire du Châtelet à Paris où il était de passage rue de Bussy (Faubourg Saint-Germain), vraisemblablement pour affaires. Il confère à sa femme tout pouvoir concernant les affaires du couple. Il est à noter qu'aucun contrôle n'est exercé de la part des deux frères de Jean, alors en activité à cette époque. Mais borner le rôle des femmes à celui de joker de luxe pour leur époux est dans le cas du commerce manceau une erreur. Il apparaît bien que les femmes avaient un rôle dans la tenue des livres de

58. CAILly, Claude, "Contribution à la définition d'un mode de production proto-industriel ", Histoire \& Mesure, vol. 8, n 1-2, 1993, p. 22.

59. BERG, Maxine, The age of manufacture 1700-1820, New-York-Oxford, Oxford University Press, 1986, p. 136-165.

60. Médiathèque Louis Aragon, Le Mans, Archives de la société d'agriculture. Mémoire anonyme v. 1790 .

61. Guicheteau, Samuel, "Y avait-il des ouvrières à Nantes au tournant des XVIII et XIX ${ }^{\mathrm{e}}$ siècles?", Annales de Bretagne et des Pays de l'Ouest, nº 114-3, 2007, p. 95.

62. Arch. dép. de la Sarthe, 111 AC 544, 1760.

63. RugGiu, François Joseph, L'individu et la famille dans les sociétés urbaines anglaise et française (1720-1780), Paris, PUPS, 2007, p. 274.

64. Arch. dép. de la Sarthe, 28 J 358, 15 mai 1739. 
comptes ${ }^{65}$, comme le montre le cas de la fille aînée de Guillaume Véron le jeune, qui visiblement avait été commis dans la boutique familiale ${ }^{66}$. D'ailleurs, la gestion des boutiques, stocks et achats de marchandises pouvait être exclusivement féminine comme le montre le cas de la femme du négociant Cureau ${ }^{67}$. De plus, on trouve une clause dans les statuts de la communauté interdisant aux femmes d'ouvrir une seconde boutique, stratégie utilisée afin de multiplier les points de vente dans la ville ${ }^{68}$. L'étendue des compétences féminines dans le commerce de l'étamine du Mans est donc extrêmement vaste et fréquente. Il conviendrait d'ailleurs d'étudier plus en détail les négociantes mancelles du XVIII ${ }^{\mathrm{e}}$ siècle.

Un élément supplémentaire démontrant cette implication peut résider dans le fait que bien souvent, à la mort de leur "moitié ", les négociants se retiraient du commerce. Par exemple, Véron-Duverger, qui à la mort d'Anne Plumard sa femme, le 20 mars 1726, se retrouva veuf pour une période de dix ans jusqu'à son remariage avec Marie-Renée Godard de la Gracinière ${ }^{69}$. Leprince d'Ardenay, un marchand cirier de la région du Mans, nous rapporte dans ses mémoires quelques éléments intéressants sur la vie de son glorieux aïeul (l'oncle de sa femme). Il signale notamment que la mort d'Anne Plumard plongea François Louis dans un profond désarroi : " il se retira peu a peu du commerce pour se livrer tout entier au goût qui le dominoit ${ }^{70}$ ". Il parle ici de son goût pour l'agriculture et les affaires publiques. Un second exemple est encore plus criant. Il concerne un des débiteurs des Véron, également fidèle partenaire commercial, le sieur Thomas Billon. Ce dernier doit surmonter le décès de sa femme en 1721 et demande un inventaire afin d'y voir plus clair dans la gestion de son commerce, "le deces d'Anne Caillere son épouse etant arrivé depuis un mois sans auparavant luy avoir donné aucune connaissance des affaires de sa maison et de son commerce dont et du tout elle avoit seule le maniement ${ }^{71}$ ". On peut dès lors voir dans les archives fiscales de la communauté des marchands drapiers le déclassement économique de cet homme qui a, semble-t-il, perdu une épouse mais aussi une partenaire commerciale de premier ordre.

\section{Vers un cartel Véron?}

Si le couple entrepreneurial revêt une importance capitale, il n'en demeure pas moins que la famille élargie prend la part du lion dans le développement commercial. D'ailleurs, les années 1740 voient les trois frères

65. Chassagne, Serge, Oberkampf..., op. cit., p. 76.

66. Arch. dép. de la Sarthe, 28 J 358, 23 octobre 1744.

67. Arch. dép. de l'Indre-et-Loire, C 116, 22 août 1735.

68. Ibid., Statuts et règlements de la communauté des marchands drapiers-merciers unis de la ville du Mans, p. 12.

69. FLEURY, Gabriel, François Veron de Forbonnais..., op. cit., p. 19-20.

70. HuBert, Benoît, Mémoires de Jean-Baptiste Henri Michel Leprince d'Ardenay (17371819) : approche d'un notable manceau au siècle des Lumières, thèse de doctorat sous la direction d'Anne Fillon, université du Maine, 2006, p. 181.

71. Arch. dép. de la Sarthe, 4 E 25 78, 27 mai 1721. 
Véron aux affaires. Le modèle qui semble dominer en ce qui concerne les alliances familiales, outre les alliances et accords coutumiers ne passant pas devant notaire, est la constitution de sociétés. On en trouve plusieurs mentions dans les inventaires après décès. Le sieur Barbet Desgranges, gendre de Guillaume Véron le jeune, est devenu d'ailleurs l'associé de sa belle-mère (Hélène Buon) à la mort de Guillaume Véron le jeune. Il est fait à plusieurs reprises mention de la société conclue entre le sieur Desgranges et Hélène Buon, une première datant du 20 octobre 1742 et une seconde du $1^{\text {er }}$ novembre 1743 avec un écrit double entre les deux contractants, à savoir le sieur et dame Desgranges et Hélène Buon au sujet de l'apprêt des étamines ${ }^{72}$. On trouve par exemple trace d'une société familiale conclue entre les frères Véron : Guillaume le jeune, François Louis Duverger et Jean de la Croix en juillet 1722. Ces accords ne semblent pas avoir été passés devant notaire. La plupart de ces arrangements familiaux doivent par conséquent nous échapper. L'année suivante, les deux frères entrèrent de nouveau en société mais cette fois en incluant deux autres membres extérieurs au cercle de proches : les sieurs Bezard et Guesné. Cette société devait être particulièrement active puisque la liasse contenant les pièces relatives à cette alliance comporte 37 actes. Nous supposons que cette société avait pour objet le commerce de l'étamine.

Ce ne fut pas toujours le cas, puisqu'en 1737 une importante société était créée pour le commerce des bougrans entre Guillaume Véron, Pierre Leurard et René Legra. Ce genre de regroupement permettait bien sûr une synergie : les marchands pouvaient se répartir les tâches pour mettre à profit les réseaux commerciaux de chacun. Ainsi, on multipliait les débouchés, les capitaux, les compétences. La stratégie mise en place par Guillaume Véron le jeune est à cet égard fort intéressante. On a pu retrouver les lettres de maîtrise de deux de ses fils : le 23 septembre 1737 (au moment où la société pour le commerce du bougran est réalisée, ce qui n'est sans doute pas une coïncidence) Guillaume Véron du Mesnil est reçu maître teinturier au Mans. Le 9 avril 1742, son frère François Michel Véron est quant à lui reçu maître dans la communauté des marchands drapiers merciers de la même ville du Mans. On a donc ici une spécialisation familiale avec un enjeu stratégique évident afin de contrôler l'intégralité du processus de production. Une spécialisation d'autant plus intéressante que la teinture représentait l'étape la plus coûteuse dans la fabrication des étamines. On peut ici parler de concentration verticale de la production.

Nous sommes alors à un moment où les Véron dominaient le commerce manceau. Par le jeu des alliances il existait " neuf à dix [familles] qui [faisaient] commerce d'Etamine, scavoir les sieurs Cureau, Veron, Desgranges, Montarou, Garnier, Boüet, Freart et Bodier ${ }^{73}$ ". On observe le même phénomène dans les grandes places marchandes comme Saint-Malo où les stratégies matrimoniales contribuent à la formation dans la première moitié

72. Arch. dép. de la Sarthe, 28 J 358, 23 octobre 1744.

73. Arch. dép. de la Sarthe, 111 AC 544, 2 juin 1747. 
du XVIII ${ }^{\mathrm{e}}$ siècle d'un patriciat urbain composé de 20 à 30 familles $^{74}$. La fratrie se retrouve alors à la tête d'une organisation pyramidale dans laquelle on trouve toutes les professions liées à la fabrication et au commerce de l'étamine. Ce cercle de fidèles est visible dans les signatures que l'on peut lire au bas du contrat de mariage de François Louis Véron Duverger et son épouse Anne Plumard en juillet $1721^{75}$. On y trouve les familles de négociants Plumard, Godard, Nouet, mais aussi les marchands Champion, Goupillau, Touzé, Pasquier, les maîtres fabricants Rousseau, Girard, Le Roy, Aubry, Labrosse ${ }^{76}$, sans oublier les marchands ciriers Orry et Le Romain. On voit alors se dessiner une hiérarchie sociale qui se manifeste d'ailleurs physiquement puisque nous trouvons dans un premier temps les signatures des proches, négociants, marchands ciriers et autres personnages d'importance majeure, les marchands alliés avant de terminer par les fabricants.

D'après les dires des fabricants, cette situation de domination des marchands drapiers est le fait de la concertation entre la dizaine de familles de grands négociants en étamine. Une situation d'entente est ici plus lucrative pour cette dizaine de familles mais réduit le bien-être total de la communauté puisqu'ils accaparent le profit des concurrents en jouant sur les prix comme le prouve cette autre plainte des fabricants datant de $17499^{77}$ : " depuis 25 à 30 ans les marchands d'étamines de la ville du Mans, avoient travaillé sans relâche pour se rendre les maîtres du prix des étamines". Cet élément est d'autant plus intéressant qu'une situation d'entente est davantage tenable si elle se prolonge dans le temps. En effet, si des firmes se partagent un marché en se mettant d'accord sur les prix (en augmentant simultanément leurs prix de vente), et qu'elles savent que les acteurs seront les mêmes pendant une période importante - ce qui est le cas sur le marché des étamines - alors elles n'ont pas intérêt à dévier de la politique d'entente car elles sont sûres de se voir punir par les concurrentes (guerre des prix, guerre commerciale, répression violente...). En revanche, si l'entente concerne une courte période, il est facile de dévier puisqu'on ne craint pas la sanction des concurrents dans le futur. Ici, la mise en avant de la stratégie progressive sur une trentaine d'années nous indique que l'entente, entre les dix familles devait être particulièrement tenable et avantageuse.

Mais nous sommes loin de l'entente cordiale : des jalousies et résistances apparurent de la part d'un clan rival de marchands cristallisés autour du sieur Cureau, notamment au travers de l'investissement dans la communauté de métier. Il arrive souvent que les formes corporatives soient investies par certains marchands et servent, in fine, leurs intérêts particuliers $^{78}$. Comme les Véron furent entre 1725 et 1750 de nombreuses fois garde juré de la communauté, on peut donc voir dans cette responsabilité le symbole de leur influence. Mais ne nous y trompons pas, il s'agit

74. Lespagnol, André, “Messieurs de Saint-Malo »..., op. cit., p. 763.

75. BnF, fond Chappée, 121, 12 juillet 1721.

76. Arch. dép. de la Sarthe, 111 AC 561, 17 août 1740.

77. Arch. dép. de l'Indre-et-Loire, C 154, 7 décembre 1749.

78. KaPlan, Steven, MinARD, Philippe, La France malade du corporatisme?..., op. cit., p. 48. 
également là d'un moyen de lutter contre leurs rivaux. On voit bien ici que le contrôle du bureau de marque est un enjeu de la plus haute importance pour les marchands : les horaires d'ouverture de celui-ci ou bien la question des droits de visite dans les ateliers des fabricants, la marque des draps ${ }^{79}$ sont bien souvent l'objet de luttes et de contestations. Ceci nous fera dire que les Véron, du fait de leur implication dans la communauté, utilisèrent cette dernière comme une arme commerciale contre leurs confrères et les fabricants. L'opposition systématique du clan Cureau nous laisse bien voir que les Véron, sous couvert de l'intérêt public, tiennent les rênes du commerce local, n'hésitant pas à user de moyens parfois proches du sabotage tels que la fermeture du bureau de marque ou bien encore le refus de marquer les draps de leurs concurrents comme s'en plaindra la femme du sieur Cureau le 22 août $1735^{80}$.

\section{Du négociant en étamine au grand notable}

\section{Les stratégies de la domination}

On l'aura compris, les Véron connurent une ascension économique rapide. Mais pour durer dans le secteur d'activité textile, il faut parfois user de stratégies. Une première option consiste à fuir l'espace réglementaire $^{81}$, en faisant fabriquer à la campagne. Il est d'ailleurs assez courant de voir les différents acteurs jouer sur les discordances entre des normes urbaines et rurales $^{82}$. Ceci est par exemple visible dans les années 1740 lorsque les maîtres fabricants du bourg de Changé se plaignent auprès de l'intendant de la généralité de Tours d'abus commis dans le contrôle exercé de la part des maîtres de la communauté des fabricants du Mans. On voit ici toutes les tensions entre ville et campagne puisque les maîtres dénoncent la violence des visites de contrôle et l'esprit des gardes qui est, selon eux, contraire à celui des règlements. Les fabricants ruraux citent le cas de la dernière visite du mois de juin au cours de laquelle les gardes " ont exigé de chaque boutique des supliants une somme de $10 \mathrm{~s}$ quils ont ensuite depensée aux cabarets avec leurs amis, apres avoir vomi toutes sortes dinvectives aux maitres ${ }^{83}$ ". Ils réclament donc à l'intendant la création d'une jurande dans le bourg de Changé afin de se soustraire à la mauvaise influence des fabricants manceaux. On voit clairement avec cet exemple une illustration des tensions qui peuvent régner entre les fabricants ruraux et ceux de la ville. Mais si pour les fabricants changéens c'est la jalousie qui guidait la besogne des gardes jurés manceaux, il semble que l'enjeu soit pour eux d'uniformiser les contrôles afin de diminuer les fraudes qui se perpétuaient à la campagne.

79. Arch. dép. de la Sarthe, 111 AC 544, 2 juin 1747.

80. Arch. dép. de l'Indre-et-Loire, C 116, 22 août 1735.

81. Minovez, Jean-Michel, L'industrie invisible..., op. cit., p. 110-111.

82. KaPlan, Steven, MinaRD, Philippe, La France malade du corporatisme?, op. cit., p. 44.

83. Arch. dép. de l'Indre-et-Loire, C 116, années 1740. 
Nous sommes donc dans une situation où les négociants falsifient littéralement les produits afin de baisser les coûts de production et pratiquer ainsi à l'encontre de leurs concurrents une véritable guerre des prix. Ceci ne peut que nous faire penser au célèbre article de G. Akerlof ${ }^{84}$ datant de 1970. L'économiste y décrivait une situation où l'acheteur était dans l'incapacité de différencier un bon produit d'un "lemon ", un mauvais produit. Il prend l'exemple du marché des automobiles d'occasion afin de mettre en évidence une situation d'asymétrie d'informations. Dans les cas des étamines nous sommes largement dans ce cadre d'analyse puisque le vendeur, le négociant, connaît la véritable qualité de son produit, alors que l'acheteur ne la connaît pas. Les conséquences que nous en tirons sont donc logiquement les mêmes. En effet, puisque les négociants sont seuls à savoir si leurs étamines sont des " lemons ", c'est-à-dire des produits défectueux, ils peuvent adapter leur prix de vente. Un marchand de "lemons" va être plus disposé à diminuer son prix, alors qu'un négociant qui vend des produits de qualité sera intransigeant sur le prix de vente. L'acheteur ira naturellement vers le produit le moins cher puisqu'il sait qu'il risque de tomber sur une pièce défectueuse quoi qu'il arrive. Il en résulte que le marché est inondé à terme de "lemons " puisque les bons produits, du fait de leur prix, ne trouvent jamais preneurs ${ }^{85}$. Nous sommes ici, exactement dans la situation que nous décrivent les fabricants dans les années 1750. Ces exemples sont fort révélateurs de la volonté des marchands et négociants de jouer sur les règles. Bien sûr, les règlements poussant les négociants à proposer des produits très similaires, la fraude est d'autant plus intéressante pour les marchands que le prix va être le principal critère de choix. Nous en déduisons que paradoxalement, plus le contrôle des étoffes est fort, plus l'homogénéité des produits est grande, plus la fraude des négociants devient profitable.

Une seconde façon de contourner les règles établies concernant le commerce des étamines est de créer un nouveau marché. On pourrait arguer que la production de bougran se développa pour compenser un marché des étamines déclinant, mais c'est en 1736 à un moment où le commerce de l'étamine est florissant que se développa cette production nouvelle. Il s'agit d'une étoffe plus grossière que l'étamine, une toile de chanvre souvent composée de restes de toile. On trouve à l'origine de ce commerce trois marchands : Pierre Levrard, Pierre Le Gras et Guillaume Véron le jeune. La manufacture s'effectuait chez le " sieur Véron rue des quatre roues où l'on avoit bâti un magazin, et un lieu propre pour travailler ${ }^{86}$ ". Un mémoire, conservé dans les archives de la généralité de Tours, sur la manufacture du Mans ${ }^{87}$ nous indique qu'il n'y avait au Mans que trois fabricants établis

84. AKERLOF, George, "The market for "lemons" : Quality, Uncertainty and the Market Mechanism ", Quarterly Journal of Economics, 1970, vol. lxxxiv, p. 488-500.

85. MinARD, Philippe, La fortune du colbertisme..., op. cit., p. 160.

86. Médiathèque Louis Aragon Le Mans : Mémoires de Négrier de la Crochardière, t. II, p. 191.

87. Arch. dép. de l'Indre-et-Loire, C 130, 30 juin 1740. 
respectivement en 1737, 1738 et 1739. En 1740, L'inventaire imprimé de la série $\mathrm{C}$ des $\mathrm{AD}$ Indre-et-Loire indique qu'en 1740 la production était de " 7 à 800 pièces de bougrans par an ". Nous apprenons également qu'il n'y avait aucun statut concernant la fabrication de ces draps. Cela faisait de ce commerce une aubaine pour Guillaume Véron qui avait seulement deux concurrents et pouvait effectuer l'ensemble de la production comme il l'entendait. Mais il convient de remarquer que le marché des bougrans ne constitua pas longtemps une " niche " pour Guillaume Véron le jeune. En effet, on n'échappe pas longtemps aux règles des communautés dans la France d'Ancien Régime. Si en 1744 on nous disait que « les personnes qui fabriquent, teignent et aprestent les Bougrans au Mans ne font aucun corps de communauté ${ }^{88}$ ", les fabricants de bougrans étaient en réalité soumis à un contrôle de la part de la communauté des marchands drapiers merciers unis depuis 1740. Une ordonnance du 3 octobre $1740^{89}$ de Charles Nicolas Le Clerc de Lesseville, maître des requêtes, intendant en la généralité de Tours, nous informe de dispositions prises pour lutter contre les abus se trouvant dans la fabrique de bougrans. Nous avons, quatre ans après le début de la fabrication, une première réglementation.

Enfin, si le fraudeur se fait prendre ou bien s'il existe un litige commercial, la discrétion est parfois de mise dans le monde du commerce. Il semble qu'une des stratégies les plus efficaces pour échapper à la justice soit de feindre une absence. Nous pouvons voir Véron de la Croix déplorer en 1750 que « le S. Cureau outre cela affecte toujours son abscence loin assure même que ce sera pour plusieurs année en continue cependant à agraver de plus an plus les coups quon a porter a ma reputation en distribuant tous les jours dans le public dans l'étranger et jusque dans les foires les monuments ineffasable de ma diffamation ${ }^{90}$ ". Déjà en 1735 , alors que le sieur Cureau est en déplacement à Marseille pour une durée indéterminée, c'est sa femme qui écrivait à l'intendant afin d'excuser son époux pour son absence ${ }^{91}$. Nous sommes ici face à une stratégie qui semble très couramment utilisée par les négociants afin de tirer profit des lenteurs de la justice et des distances importantes entre négociants. Ceci rendait le contrôle d'autant plus difficile.

\section{L'entrepreneur «prédateur» ${ }^{92}$}

Nous voyons donc ici apparaître au travers des différentes stratégies évoquées ci-dessus la figure d'un entrepreneur prédateur. C'est d'ailleurs ce qui lui est principalement reproché. Les principaux arguments que les marchands et fabricants objectèrent à Guillaume Véron en 1712, lors de la

88. Ibid., 28 juin 1744.

89. Ibid., 3 octobre 1740.

90. Ibid., 18 octobre 1750 .

91. Ibid., 12 novembre 1750 .

92. Vullermot, Catherine, Villette, Michel, Portrait de l'homme d'affaires en prédateur, Paris, La découverte, 2005. 
transmission du privilège à ses enfants, nous fournissent un bon exemple de l'opinion que l'on avait de cette famille. On voit alors apparaître la figure d'un entrepreneur prédateur, guidé par l'ambition. On trouve d'ailleurs à sept reprises la mention de l'ambition des Véron. Cette ambition démesurée est fréquemment opposée à l'intérêt général, ce qui va dans le sens de ce que les autorités veulent entendre, et entre bien sûr dans le cadre d'une rhétorique juridique qui ne doit pas nous induire en erreur.

Il en va de même pour les arguments employés à l'encontre des négociants lors d'un procès en 1757 visant à interdire l'accès à la ville du Mans aux marchands forains. Toutefois, le discours tenu est moins hostile aux Véron qu'à la caste des négociants qui domine l'économie de la ville. Cette fois, on est moins dans la dénonciation de la nuisance au bien public, et davantage dans la condamnation de l'immoralité qu'est la réalisation de tels profits au détriment des autres marchands. Une opposition plus sociale à l'intérieur du groupe des marchands apparaît ici. On peut alors voir poindre un discours sur la cupidité et la jalousie des marchands qui veulent accaparer l'ensemble des richesses. Frédérique Pitou remarque de plus que ce type d'argumentaire revient régulièrement dans le Maine à cette période à l'encontre des élites urbaines ${ }^{93}$. Il résulte d'une incompatibilité entre, d'une part, la morale marchande qui exalte la richesse et, d'autre part, les valeurs des élites nobiliaires. Ce qui explique que les deux groupes restent distincts dans la plupart des villes ${ }^{94}$.

\section{Un retrait progressif $\mathrm{du}$ commerce}

Il est courant que la deuxième génération d'entrepreneurs abandonne le commerce pour se tourner vers des tâches touchant à la vie publique ${ }^{95}$. Il en va de même dans le Midi toulousain où Jean-Michel Minovez constate que les entrepreneurs de l'industrie textile se tournent $\mathrm{au} \mathrm{XVIII}^{\mathrm{e}}$ siècle vers d'autres activités, une fois fortune faite ${ }^{96}$. Toutefois cette reconnaissance sociale fut relativement précoce pour cette famille puisque Guillaume Véron l'aîné fut procureur de la fabrique de la paroisse du Pré, premier juge de la juridiction consulaire mais aussi gardien du grenier à sel de la ville du Mans. Ses fils eurent également un rôle à jouer dans la vie de la cité mancelle. C'est en 1710 qu'est fondée la juridiction consulaire du Mans par un édit de Louis XIV. Il s'agit d'un tribunal de commerce ayant pour fonction de régler de façon rapide les différends entre marchands ou négociants. On compte 67 juridictions consulaires à l'aube de la Révolution de

93. Pitou, Frédérique, "La richesse discrète des élites du Maine ", dans : BouRQuin, Laurent, HAMON, Philippe, Fortunes urbaines élites et richesses dans les villes de l'Ouest à l'époque moderne, Rennes, PUR, 2011, p. 69-71.

94. Ibid., p. 59.

95. MARCHESNAY, Michel, "L'entrepreneur : une histoire française ", Revue française de gestion, $\mathrm{n}^{\circ}$ 188-189, 2008, p. 77-95.

96. MinOvEZ, Jean-Michel, « Les manufactures royales de draps fins du Midi toulousain et leurs entrepreneurs au XVIII siècle ", Annales du Midi : revue archéologique, historique et philologique de la France méridionale, t. 112, n 229, 2000, p. 32. 
$1789^{97}$. Au Mans, il fallait pour y entrer avoir plus de 27 ans et posséder une expérience particulièrement importante dans le domaine du commerce afin de pouvoir démêler les affaires les plus complexes. Cela suppose donc, par la même occasion, une bonne connaissance des textes de lois et des règles du commerce ${ }^{98}$. Véron-Duverger fut également appelé à la juridiction consulaire, comme son père, en 1745 et 1762 où il fut nommé consul puis doyen. ${ }^{99}$ Guillaume Véron l'aîné fut lui aussi appelé aux responsabilités en tant qu'échevin en 1711, tout comme son fils Guillaume Véron le jeune en 1726, et Véron-Duverger, lui aussi échevin de la ville du Mans en 1754. Bien sûr, Véron-Duverger sera en 1761 le premier président du bureau d'agriculture du Mans, émanation de la Société royale d'Agriculture de la généralité de Tours. Ce poste lui permet de devenir une figure de l'agronomie dans le haut Maine.

Toutes ces fonctions témoignent donc d'un intérêt pour la vie publique et d'un besoin de reconnaissance sociale de la part des élites négociantes. André Lespagnol observe d'ailleurs le même phénomène à Saint-Malo ${ }^{100}$. C'est certainement une façon de légitimer leur fortune. Olivier Chaline note d'ailleurs l'importance du paraître dans la classe des marchands rouennais à la même époque ${ }^{101}$. Au Mans, cette classe de marchands semble également aspirer à vivre noblement, à rechercher les honneurs sur le plan local. Si l'aristocratie mancelle n'est pas bâtisseuse, les Véron aiment à investir dans des terres à la campagne (Saint-Mars d'Outillé, Bonnétable...) où ils pratiquent la chasse. Sur la période étudiée, la famille ne semble pas accéder à la noblesse, à l'inverse de la plupart des marchands de Saint-Malo et de certains grands négociants manceaux. En effet, la cité malouine avait en 1715 une part non négligeable de marchands devenus nobles, celleci s'élevant à $62 \%{ }^{102}$. Pour Le Mans, Benoît Hubert évoque une liste de marchands devenus nobles tels que les Cureau ou encore les Plumard ${ }^{103}$. François Véron de Forbonnais est, quant à lui, élu député de la noblesse en 1789, mais son élection pose question puisque ses lettres de noblesse sont très récentes ${ }^{104}$. Nous pouvons donc penser que ses ancêtres n'étaient pas nobles et que ses lettres d'anoblissement viennent récompenser des années d'aspiration au second ordre ${ }^{105}$.

97. Ibid., p. 120 .

98. Hubert, Benoît, Mémoires de Jean-Baptiste Henri Michel Leprince d'Ardenay..., op. cit., p. 108.

99. Pesche, Julien Rémi, Dictionnaire topographique, historique et statistique de la Sarthe : tome III, Paris, Le livre d'Histoire, 1999 (1824), p. 433.

100. Lespagnol, André, “ Messieurs de Saint-Malo »..., op. cit., p. 758

101. Chaline, Olivier, "L'argent bien tempéré. Les élites rouennaises et la visibilité de l'opulence au XVIII ${ }^{\mathrm{e}}$ siècle ", dans BouRQuin, Laurent, Hamon, Philippe, Fortunes urbaines..., op. cit., p. 48.

102. Lespagnol, André, “Messieurs de Saint-Malo »..., op. cit., p. 261.

103. Hubert, Benoît, Mémoires de Jean-Baptiste Henri Michel Leprince d'Ardenay..., op. cit., p. 335.

104. Haureau, Jean-Barthélemy, Histoire littéraire du Maine Tome IX, Genève, Slatkine Reprints, 1969 (1870-1877), p. 199.

105. LesPagnol, André, “Messieurs de Saint-Malo »..., op. cit., p. 758. 
Nous pouvons donc rejeter la vision schumpeterienne de l'entrepreneur développée par François Dornic. Le cas de Guillaume Véron l'aîné n'est pas celui d'un self made man du siècle des Lumières, inventant de nouvelles techniques afin de briser le carcan des communautés de métier. À l'inverse, il apparaît que la famille Véron s'impliqua pleinement dans la communauté des marchands de la ville du Mans, afin d'asseoir son pouvoir et de développer une stratégie commerciale reposant à la fois sur l'obtention du privilège royal et le noyautage des institutions locales. De plus, il apparaît que l'on n'entreprend jamais vraiment seul dans le domaine du textile comme ailleurs. L'importance de la famille est telle que les négociants agissent en tandem avec leur épouse et un cercle familial élargi. Ce type d'organisation familiale permet une certaine spécialisation au sein de la cellule économique, ainsi que la baisse du risque lié aux aléas de la conjoncture économique en diversifiant les sources de revenus et les canaux commerciaux. Il convient donc de réévaluer la place des femmes dans ces entreprises commerciales. Souvent présentées comme une comptable, une intendante, gérant occasionnellement la boutique en cas d'absence du chef de famille, il semble plutôt qu'elles puissent assumer une partie de la gestion de la boutique et des relations commerciales. Ces organisations familiales, alliées à des stratégies visant à asseoir la domination du clan Véron, nous offrent une image différente de l'entrepreneur au siècle des Lumières. Toutefois, si les Véron ont réussi à gravir rapidement l'échelle sociale, le statut de marchand, si rémunérateur soit-il, ne confère pas une reconnaissance sociale suffisante. En effet, la condamnation des fortunes trop rapides rend le marchand sujet à la critique du peuple et à la jalousie de ses confrères. On voit donc poindre la figure d'un entrepreneur prédateur, mais si l'on dépasse la vision donnée par les sources judiciaires, nous pouvons également voir en eux des personnages plutôt conquérants (innovations techniques, lancement de la fabrication de bougrans, conquêtes de nouveaux marchés...). Il s'agit donc d'un statut social controversé et de manière assez classique les Véron, notamment Duverger vers 1745-1750, vont se retirer du grand commerce pour se consacrer à un rôle de notable local. 


\section{RÉSUMÉ}

Le commerce de l'étamine, une étoffe de laine précieuse, fait la renommée du Mans aux $\mathrm{XVII}^{\mathrm{e}}$ et $\mathrm{XVII}^{\mathrm{e}}$ siècles. Parmi les familles de marchand-fabricants, puis de négociants, les Véron ont une place centrale. Ils seraient à l'origine de la création de l'étamine ainsi que de sa diffusion vers de lointains marchés. Si la famille a surtout été étudiée pour l'apport de François Véron de Forbonnais à la pensée économique du XVIII siècle, l'article propose de se concentrer sur les fondateurs de cette véritable dynastie entrepreneuriale. Une relecture du cas de Guillaume Véron l'aîné, au cœur d'un procès visant à l'obtention d'un privilège d'invention (1695-1699), est ainsi proposée au prisme de l'historiographie récente. L'étude des stratégies entrepreneuriales et du rôle du cercle familial élargie, notamment aux femmes, permet de nuancer la vision d'un self made man des Lumières. Enfin, l'étude de l'entrepreneur dans son contexte social permet d'étudier la trajectoire de la famille jusqu'à son retrait progressif du commerce dans la seconde moitié du XVIII ${ }^{\mathrm{e}}$ siècle.

\section{ABSTRACT}

The trade of muslin, a precious woolen cloth, made a name for Le Mans during the XVIIth and XVIIIth centuries. Among the merchant-manufacturer families, then traders, the family of Veron played a major role. They created muslin and ensured its distribution towards foreign markets. The family has primarily been studied with regard to the legacy of François Véron de Forbonnais and to economic thought in the 18th century. In contrast, this article instead focuses on the founders of this entrepreneurial dynasty. A re-reading of the case of Guillaume Véron the elder, at the heart of a lawsuit regarding the acquisition of letters patent (1695-1699) is thus read through the prism of recent historiography. The study of entrepreneurial strategies and the role of the family circle, especially that of women, enables us to qualify the idea of a self-made man. Finally, the study of the entrepreneur in his social context allows us to chart the family's path until its gradual withdrawal from trade in the second half of the 18th century. 